\title{
Aplicación móvil basada en el contexto para promover el aprendizaje del idioma inglés
}

Context-based mobile application to encourage English language learning

Osiris Montero Ríos ${ }^{1}$

osirismonterorios@gmail.com

Francisco Aguilar Acevedo'

aguilar.afco@sandunga.unistmo.edu.mx

Guadalupe Toledo Toledo'

gtoledo_1207@hotmail.com

Silvia Reyes Jiménez ${ }^{1}$

chivisza9@sandunga.unistmo.edu.mx

Daniel Pacheco Bautista ${ }^{1}$

dpachecob@bianni.unistmo.edu.mx

IUniversidad del Istmo 
Resumen: La telefonía móvil que en un principio se comercializó como dispositivos de comunicación y entretenimiento, en la actualidad es empleada en sectores tan diversos como la educación. Dentro de los métodos de apoyo al proceso de aprendizaje destacan las aplicaciones móviles y en especial las relacionadas con los idiomas. En su mayoría, estas aplicaciones emplean estrategias de enseñanza universal que sugieren situaciones ajenas al entorno sociocultural del usuario, lo cual podría impactar en su aprendizaje. En este artículo se muestra el desarrollo de una aplicación Android para promover el aprendizaje del idioma inglés basada en información provista por el usuario que le resulta de su interés. Se presenta el diseño del contenido educativo y de la aplicación móvil que permiten al usuario generar su propio material didáctico en base a sus intereses. Como caso de estudio se considera la evaluación de la aplicación para el área de las ciencias computacionales.

Palabras clave: Sistemas basados en el contexto, Android, aprendizaje móvil.

Abstract: Mobile telephony was initially marketed as communication and entertainment devices. It is currently used in different sectors such as education. Mobile applications, especially those related to language, stand out within the methods that support learning. Most applications use universal teaching strategies and propose situations that are unrelated to the user's socio-cultural environment. This is a drawback that may affect learning. This article presents the development of an Android application for the learning of English which is based on the information provided by the user that is of interest to you. The design of both the educational content and the mobile application that allow users to generate their own learning material according to their own interests is also presented. The case study is the assessment of the application within a computer science área.

Keywords: Context-based systems, Android, m-learning 


\section{Introducción}

Saber inglés se ha convertido en una competencia básica de estudiantes de todos los niveles educativos. Pero ¿por qué es tan importante el idioma inglés? Según Calderón (2015), porque hay más de dos mil millones de personas que lo usan regularmente y además se habla en 138 países, porque 4 de cada 5 interacciones en inglés se dan entre personas para las cuales ese idioma no es su lengua materna, porque particularmente en México uno de cada dos empleos con ingreso superior a cincuenta mil pesos exige el uso constante del inglés, y porque hablar una segunda lengua tiene un impacto positivo sobre el desarrollo neurológico. Más aún, muchas de las publicaciones científicas y libros se divulgan en inglés, hecho que acentúa su importancia en la educación, la investigación y la práctica profesional. Sin embargo, en México las marcadas deficiencias en el dominio del idioma inglés, es una problemática en todos los niveles educativos la cual no es privativa de un estado o región; sino de todo el país (Lemus, Duran, y Martínez, 2008).

En respuesta a este tipo de problemáticas, organismos como la UNESCO (Organización de las Naciones Unidas para la Educación, la Ciencia y la Cultura), han fomentado iniciativas encaminadas a estudiar de qué manera las tecnologías móviles pueden propiciar la consecución de la educación para todos, señalando que el m-learning o aprendizaje móvil proporciona estrategias al proceso de enseñanza- aprendizaje a través del uso de computadoras portátiles, tabletas electrónicas, teléfonos inteligentes (smartphones) e incluso teléfonos móviles ${ }^{1}$ (UNESCO, s.f.). Dicha organización ha publicado una serie de estudios sobre aprendizaje móvil, cuyo propósito es lograr una mayor comprensión de cómo las tecnologías móviles pueden ser utilizadas para mejorar el acceso, la equidad y la calidad de la educación en todo el mundo. En dicha documentación se señala que a medida que aumenta la cantidad y el tipo de información que pueden recopilar los dispositivos móviles acerca de sus usuarios, este tipo de tecnología será más apta para la individualización del aprendizaje, otorgando a los estudiantes mayor flexibilidad para avanzar a su propio ritmo y seguir sus propios intereses, lo que podría aumentar su motivación para aprovechar las oportunidades de aprendizaje (UNESCO, 2013).

Por otra parte, el popular uso de los dispositivos móviles como smartphones y tablets, han incorporado nuevos paradigmas con el objetivo de mejorar la experiencia del usuario. Numerosas aplicaciones comerciales en las dos plataformas dominantes en el mercado: iOS y Android, son testimonio de estos nuevos paradigmas. Un enfoque para mejorar la experiencia del usuario de una aplicación es realizar un seguimiento del contexto, considerada como aquella información que se puede utilizar para caracterizar la situación de una entidad, donde una entidad puede ser una persona, un lugar u objeto (Kolangade, 2013). El desafío es identificar aquellas piezas de información contextual que son 
consideradas más relevantes y que pueden ser obtenidas de manera fiable (Peña, 2016).

Así, en este artículo se presenta el desarrollo de una aplicación para móviles Android que permite al usuario generar su propio material didáctico contextualizado a partir de textos de su interés escritos en idioma inglés, que él mismo provee, fomentando de esta manera su autoaprendizaje. Como caso de estudio se presenta la evaluación de la aplicación para lecturas acordes al tópico de las ciencias computacionales en idioma inglés, considerando que la mayoría del vocabulario técnico que se utiliza en esta área tiene su fundamento en dicho idioma. Es de resaltar que la aplicación muestra un grado de flexibilidad no observado en aplicaciones comerciales.

\section{Aplicaciones Basadas en el Contexto}

En el campo de la computación se denomina contexto a cualquier información que puede ser usada para caracterizar la situación de una entidad (persona, lugar u objeto), y que es considerada relevante para la interacción entre el usuario y la aplicación (Abowd, et al., 1999). Los sistemas que toman en cuenta el contexto en su interacción con el usuario o con la lógica de procesamiento suelen denominarse sistemas basados en el contexto.

En un sistema de cómputo, las acciones del usuario que se requieren como entrada para determinar la solución, dirección o acción, pueden ser parcial o completamente eliminadas con el uso de contextos (Kolangade, 2013). Esto es especialmente cierto en los sistemas de cómputo móvil donde la naturaleza portátil inherente de los dispositivos permite que exista un gran número de variables influyentes tales como la edad, el género, los gustos, entre otras, que pertenecen al entorno, razón por lo cual, los sistemas basados en el contexto han sido investigados y desarrollados, empleando dispositivos móviles, desde hace más de una década. Por ejemplo, en Zheng, Cheng y Xu (2016) se describe una aplicación de comercio electrónico (e-commerce) con una interfaz de usuario que se adapta en función de la información del dominio del usuario como la edad, el género, la categoría del usuario e información de los cambios en el entorno como el nivel de batería y la disposición de Wi-Fi. Un árbol de adaptación es empleado para representar la adaptación de la interfaz de usuario en el dispositivo.

Con propósitos de enseñanza-aprendizaje se han realizado diversas investigaciones. En Wanumen, Cavanzo, y Guevara (2015) se aborda el desarrollo de una plataforma de enseñanza-aprendizaje de conceptos básicos sobre astronomía a través de una aplicación Android con dos modelos de adaptación: uno basado en el perfil de usuario de Facebook y el otro con 
atributos pertenecientes al contexto. Los datos de la cuenta de Facebook brindan a la plataforma de enseñanza-aprendizaje la información necesaria para perfilar el rol al que pertenecerá el usuario (ya sea docente o estudiante básicointermedio-avanzado).

Para el caso del modelo de adaptación basado en el contexto se hace uso de la ubicación del usuario y el tiempo calendario. En el primer caso, la interacción con el usuario se limita a mostrar su cambio de ubicación mediante una brújula en pantalla e indicar cómo llegar a un determinado lugar. El tiempo calendario permite al sistema mostrar las fases de la luna y calcular su fase actual dependiendo de la época del año. Bajo la otra perspectiva, en Gómez, Hernández y Morales (2015) se presenta un sistema de aprendizaje situado capaz de entregar contenidos a estudiantes de ingeniería de acuerdo con el contexto. La arquitectura del sistema se fundamenta en la filosofía clienteservidor. El cliente se configura para proporcionar un acceso offline y online. En el caso de la configuración offline se hace uso de la realidad aumentada mediante objetos modelados en 3D que son almacenados-presentados en una computadora personal. Mediante el acceso online el estudiante puede seleccionar a través de una aplicación móvil, el tipo de lectura a realizar: QR Code (Quick Response, Código de Respuesta Rápida) o NFC (Near Field Communication, Comunicación de Campo Cercano), mientras el servidor gestiona los contenidos respectivos. Se realizan tres experiencias de aprendizaje con estudiantes de los programas de Ingeniería de Sistemas, Eléctrica y Mecánica.

En lo que respecta al aprendizaje de idiomas, en Morales, Igler, Böhm, y Chitchaipoka (2015) se presenta una aplicación móvil para el aprendizaje de idiomas como apoyo a usuarios que viven en países extranjeros. En primera instancia, la aplicación Android se enfoca en estudiantes de Alemania y Tailandia interesados en programas de intercambio entre estos dos países. La aplicación sugiere vocabulario, frases u oraciones que son útiles para el usuario, considerando atributos tales como el género del usuario, la geolocalización y el lenguaje nativo.

Los resultados reportados estiman que el enfoque propuesto mejora la experiencia de aprendizaje al considerar el contexto de uso, resultando en un proceso de aprendizaje más efectivo. Por su parte, en Sun, Hou, Hu y Al-mekhlafi (2015) se describe un prototipo de sistema para el aprendizaje del idioma Chino dirigida a extranjeros en China cuya nativa o segunda lengua es el inglés. La aplicación proporciona materiales de aprendizaje relacionados con lo que el usuario está haciendo o lo que va a hacer. El sistema propuesto presenta una arquitectura cliente-servidor vía red inalámbrica. La base de datos en el servidor almacena los materiales de aprendizaje, mientras la aplicación móvil cliente provee la interfaz de usuario. El sistema también puede adaptarse a los perfiles 
de los estudiantes como muchos sistemas de aprendizaje electrónico (elearning) existentes.

\section{Organización del Contenido Educativo de la Aplicación Propuesta}

En la Figura 1 se ilustra un proceso seguido por el usuario para solucionar su problema de aprendizaje. En búsqueda de apoyo en su proceso de aprendizaje, el usuario encuentra en la educación presencial una acertada opción cuyo costo sin embargo va en aumento (Jardines, 2010). Por otra parte, se tiene a la educación a distancia que mediante el uso de la tecnología busca maximizar la interacción profesor-alumno, enfatizando en la autonomía del estudiante. Desprendida de esta instrucción a distancia se encuentra el aprendizaje móvil, como un método de apoyo al proceso de aprendizaje mediante el uso de los ahora comunes dispositivos móviles. El elemento final de este proceso señala la atención al usuario mediante aplicaciones con estrategias de enseñanza que tienen mucho de universal, al sugerirle situaciones ajenas a su entorno sociocultural, lo cual podría impactar en su aprendizaje.

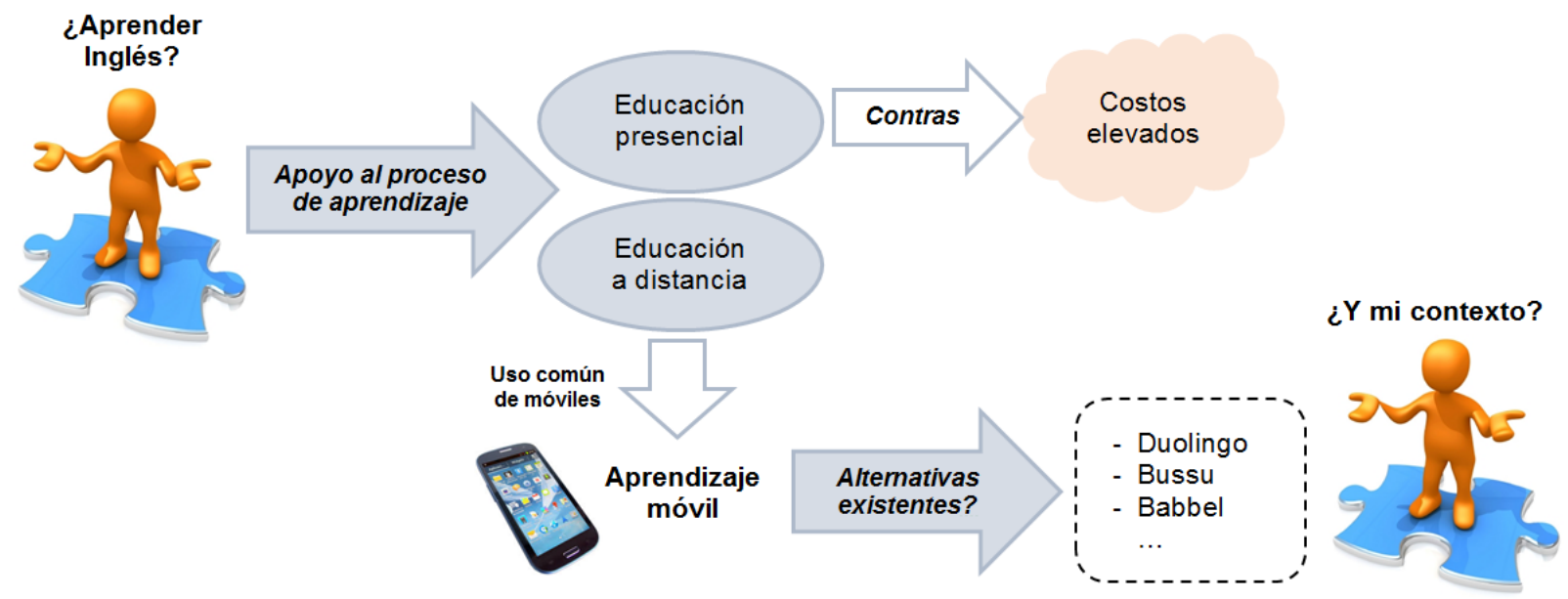

Figura 1. Necesidad del usuario.

\subsection{Contenido Educativo}

Sobre el uso y aprendizaje de lenguas, el Consejo de Europa (2002) señala: "Las personas utilizan las competencias (generales y comunicativa) que se encuentran a su disposición en distintos contextos y bajo distintas condiciones y restricciones, con el fin de realizar actividades de la lengua que conllevan procesos para producir y recibir textos (hablados o escritos) relacionados con temas en ámbitos específicos,...". En este sentido, el contexto se refiere al: "conjunto de acontecimientos y de factores situacionales (físicos y de otro tipo), tanto internos como externos a la persona, dentro del cual se producen los actos 
de comunicación". Para el caso particular de esta aplicación se plantea el contexto en términos de las lecturas en inglés que el usuario proporciona y que se antepone reflejan sus intereses, cualesquiera que estos sean, lo que le permitirá generar material didáctico personalizado.

Las competencias generales son las que no se relacionan directamente con la lengua, mientras las comunicativas son las que posibilitan a una persona actuar utilizando específicamente medios lingüísticos. La competencia comunicativa se describe como un conjunto de competencias interrelacionadas, que incluyen competencias lingüísticas, sociolingüísticas y pragmáticas, integradas con las competencias generales del individuo. Las competencias lingüísticas incluyen los conocimientos del sistema fonético, vocabulario y sintaxis de la lengua. Las competencias sociolingüísticas hacen referencia a la capacidad de una persona para producir y entender adecuadamente expresiones lingüísticas en diferentes contextos de uso. Las competencias pragmáticas tienen que ver con el uso funcional de los recursos lingüísticos sobre los escenarios de intercambios comunicativos (Consejo de Europa, 2002).

Desde el punto de vista del diseño de instrucción la aplicación presentada se desarrolla bajo un enfoque constructivista con la premisa que el conocimiento emerge en contextos que le son significativos al estudiante (Ertmer y Newby, 1993). La descomposición de textos e identificación de componentes gramaticales para generación de contenidos personalizados son la base del diseño de instrucción. Las aplicaciones para el aprendizaje de idiomas más señaladas en la web como Duolingo, Bussu y Babbel, no consideran directamente elementos que personalicen el material didáctico.

Bajo este panorama, la aplicación móvil plantea un desarrollo conjunto de las competencias lingüísticas2, sociolingüísticas y pragmáticas, para el aprendizaje de idioma inglés, a través de tres mecanismos (véase Figura 2):

1) Categorías (o partes de la oración) como ejes temáticos: conformada por la lingüísticas de las categorías léxicas (sustantivo, verbo, adjetivo, adverbio, preposición y pronombre) y funcionales o gramaticales (determinador, auxiliar, conjunciones) propias del idioma inglés (van Gelderen, 2010).

2) Información del contexto: conformada por textos en inglés referentes a los intereses del usuario.

3) Diccionario de términos: conjunto de definiciones de un contexto, como elemento de apoyo para la comprensión del texto provisto por el usuario. 


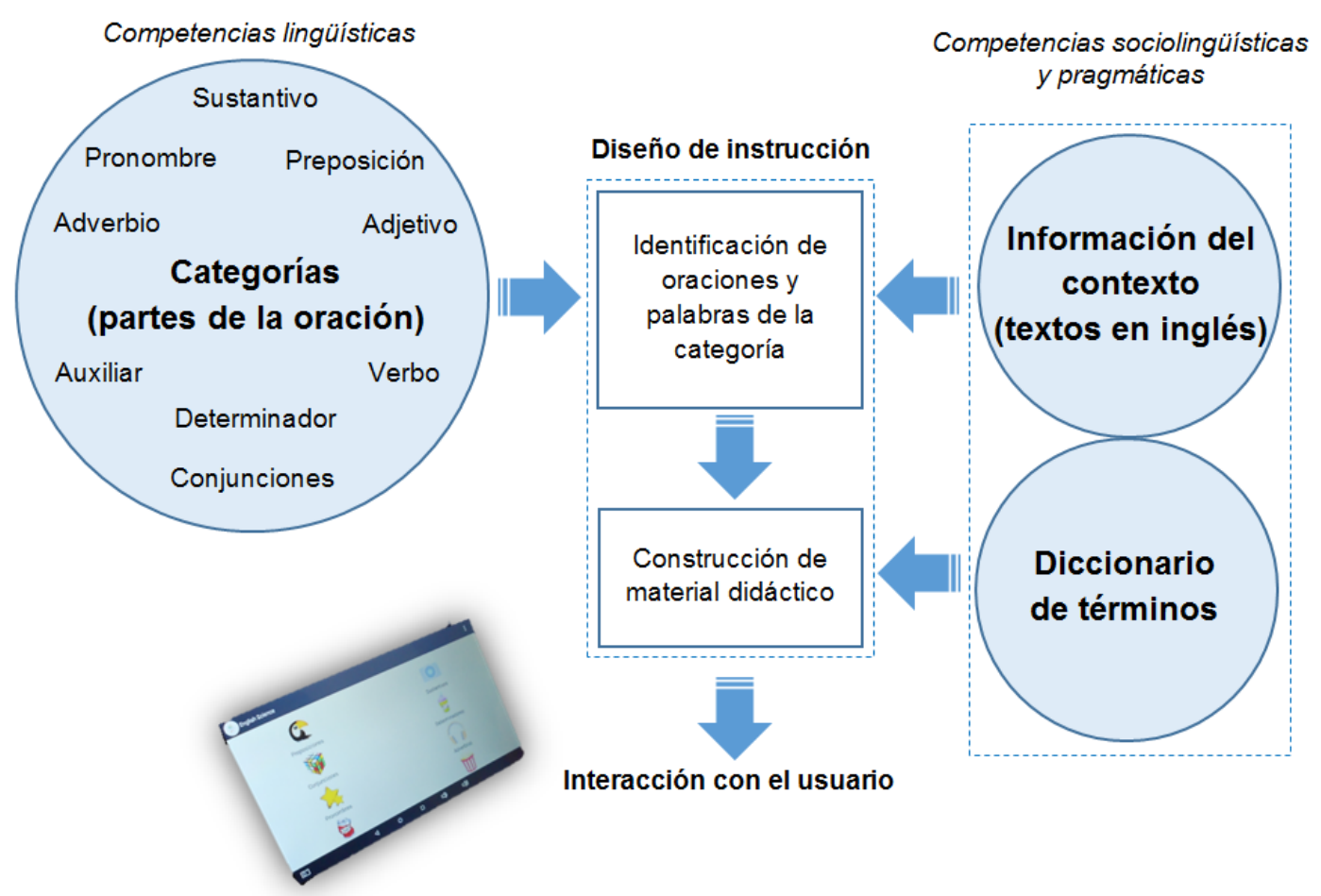

Figura 2. Diseño conceptual del contenido educativo de la aplicación.

Los materiales didácticos llamados lecciones, que se definen a través de los mecanismos listados, se componen de cuatro secciones. La lectura con el texto en inglés, el glosario con la definición de términos/palabras en el texto, la gramática con información sobre la categoría seleccionada y los ejercicios. La lectura, el glosario y la gramática forman la base de conocimiento que el usuario requiere para su aprendizaje. Los ejercicios son el medio regulador del autoaprendizaje en el usuario.

En la Figura 3, se muestra un ejemplo de un material didáctico, bajo la categoría de adverbios como eje temático, en el que puede apreciarse la integración de los conceptos descritos anteriormente. En este caso, la lectura de interés que el usuario proporciona, corresponde a un texto sobre generalidades de la programación. Ya dentro de la aplicación, una vez cargada una lectura es posible desarrollar ejercicios para diferentes categorías. 


\section{PROGRAMACIÓN}

In use today are more than a bilion general-purpose computers and bilions more cell phones, smartphones and handheld devices (such as tablet computers). According to a study by eMarketer, the number of mobile Internet users will reach approximately $134 \mathrm{mili}$ ion by 2013 . Other sudies have projected smartphone sales to surpass personal computer sales in 2011 and tablet sales to account for over $20 \%$ of all personal computer sales by 2015. By 2014, the smatphone applications maket is expected to exceed $\$ 40$ bilion, which is creating significant opportunities for programming moble applications.

(Deitel y Deitel, 2012)

\section{Glosario}

cell phones Teléfono portátil sin hilos conectado a una red celular y que permite al usuario su empleo en cualquier lugar cubierto por la red (Femández, 2011).

Intemet Red de telecomunicaciones nacida en 1969 en los EE.UU. a la cual están conectadas centenares de millones de personas, organismos y empresas en todo el mundo (Femández, 2011).

personal computer Una computadora diseñada para ser utilizado por una sola persona a la vez. (Microsoft, 2002).

application Un programa informático que lleva a cabo una función con el objeto de ayudar a un usuario a realizar una determinada actividad (Femández, 2011).

programming El arte y ciencia de la creación de programas de computadora (Microsoft, 2002).

tablet computers Un tipo de computadora portátil que tiene una pantalla LCD en la que se puede escribir con un lápiz 0 un boligrafo digital. La pantalla se puede plegar fácilmente o girar (Remacha, 2008).

\section{Gramática: Adverbios}

Los adverbios son palabras que dicen más sobre los verbos, adjetivos y otros adverbios. Muchos adverbios son construidos agregando la terminación mente (ly) a los adjetivos. Algunas palabras que terminan en mente no son adverbios. Algunos adjetivos terminan también en ly (ej Sam was feeling very lonely.)

Adverbios de tiempo: describen cuando algo sucede, responden a la pregunta "Cuándo?" (often, always, sometimes, early, late, again, yesterday, today, tomorrow,...).

Adverbios de modo describen la forma en que se hace algo, responden a la pregunta "Cómo?" (clearly, correctly, carefully. smartly,....).

Adverbios de lugar indican dónde pasa algo, responden a la pregunta "Dónde?' (outside, upstairs, here, there, anywhere, away...)

Elemplos: $\quad$ He often swims in the evening.

He was driving carefully.

Come here!

Algunos otros adverbios: here, however, more, once, quickly, there,

\section{Ejercicios}

Llene el espacio en blanco con la palabra correcta

1. In use ___ are ___ than a billion general-purpose computers and bilions tablet computers). hancheld devices ( eMarketer, the number of mobile Intemet users will reach cell phones, smartphones and

2. According to a study all personal computer sales 134 milion _ 2013.

3. Other studies have projected smartphone sales to surpass personal computer sales in 2011 and tablet sales to account for over $20 \%$ of all personal computer sales 2015.

4. 2014, the smartphone applications market is expected to exceed $\$ 40$ bilion, which is creating significant opportunities for programming mobile applications.

Figura 3. Ejemplo de material didáctico. 


\section{Diseño Funcional de la Aplicación}

En una aplicación, la arquitectura de la información permite organizar el contenido y funciones de la misma, así como estudiar su complejidad, además, nos permite analizar los diferentes niveles de profundidad, visualizar y entender la relación entre contenidos de una manera organizada (Cuello y Vittone, 2013). En la Figura 4 se presenta la arquitectura para la aplicación propuesta en función de la organización del contenido educativo.

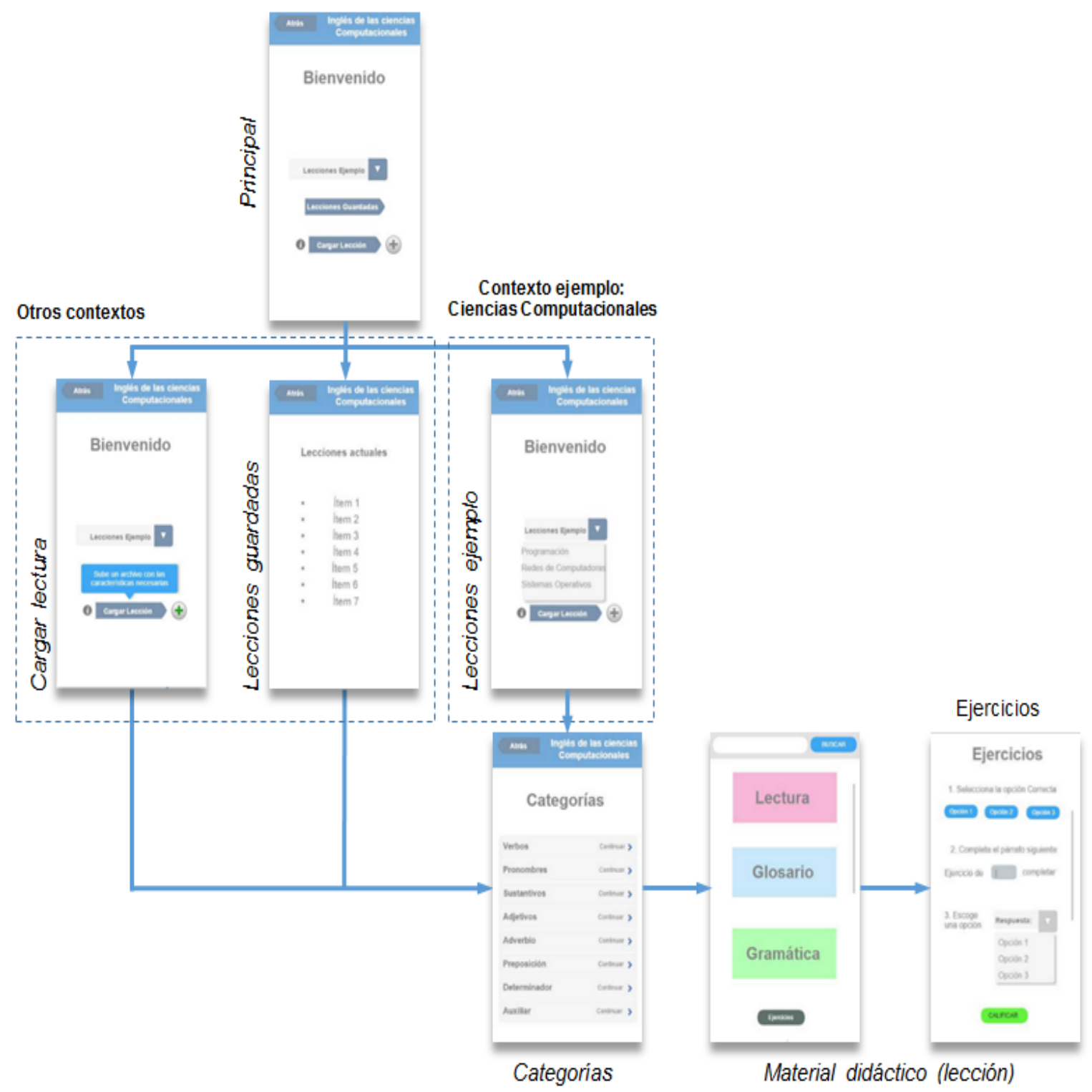

Figura 4. Arquitectura de información para la aplicación.

La pantalla Cargar lectura permitirá al usuario ingresar nueva información proveniente de temas de su interés. La pantalla Lecciones guardadas facilita el uso de información ya incorporada a través de la pantalla Cargar lectura. La 
pantalla Lecciones ejemplo muestra un menú de temáticas alrededor del ámbito de las ciencias computacionales, y que representan el caso de estudio desglosado en la sección 6. De manera puntual, las pantallas en este nivel permiten ingresar nueva información de interés del usuario (Cargar lectura), recuperar textos ya utilizados (Lecciones guardadas) y hacer uso de información precargada en la aplicación (Lecciones ejemplo). Partiendo de cualquiera de las pantallas mencionadas, se observará un listado de las categorías a seleccionar. Realizada la elección se desplegará el material didáctico generado a partir de la información proporcionada y la categoría elegida las cuales se han descrito en la sección 3.1. La lección es mostrada a través de dos pantallas, la primera con la lectura, el glosario y la gramática y una segunda con ejercicios de opción múltiple donde un registro de aciertos y errores permiten al usuario la regulación de su autoaprendizaje. Para generar material didáctico de otra categoría, basta con regresar a la pantalla Categorías y realizar una nueva selección.

Para adherir información de interés del usuario, a través de la pantalla Cargar lección, el usuario deberá seleccionar un archivo de texto (con un máximo de 1700 caracteres/ una cuartilla) almacenado en su dispositivo móvil, para lo cual deberá contar con un explorador de archivos. La aplicación almacena la información incorporada en archivos de texto no visibles al usuario. En caso de no contar con una aplicación de este género, existen diversas alternativas tales como: ES Explorador de Archivos, File Explorer, File Wrangler, Root Browser, Root Explorer, ASTRO Administrador de Archivo y AntTek Explorer Ex. Todas estas aplicaciones de distribución gratuita pueden ser descargadas a través de la plataforma Google Play Store.

\section{Desarrollo de la Aplicación}

Las tareas de programación se desarrollaron bajo una plataforma Android, empleando el kit de desarrollo de Java (JDK, Java Development Kit), el entorno Android Studio 1.3 y el kit de desarrollo de software de Android (Android SDK tools). Se empleó un smartphone modelo BLU Dash JR, con pantalla de 3.5" y sistema operativo Android v4.4 KitKat, durante las pruebas funcionales de la aplicación.

En la Figura 5 se muestra el flujo de información a través de los diferentes niveles marcados por la arquitectura de la aplicación. Para generar el glosario y los ejercicios del material didáctico (lección) se realizaron tres búsquedas secuenciales de texto empleando algoritmos de fuerza bruta (Stephens, 2013). La primera corresponde a la búsqueda sobre el texto en inglés del carácter patrón “.”, que permite separar las oraciones. Una vez identificadas las oraciones se realiza una segunda búsqueda donde los patrones corresponderán a las palabras disponibles en la base de datos de categorías (de acuerdo a aquella seleccionada). Esta búsqueda dará como resultado la información que compone 
los ejercicios, donde cada uno de ellos será una oración que contiene algún elemento de la categoría seleccionada. Finalmente utilizando las palabras encontradas se ejecuta una tercera búsqueda ahora sobre la base de datos de términos, lo cual permitirá definir el glosario de las palabras encontradas en el texto.

La base de datos asociada al diccionario de términos se encuentra limitada al contexto del caso de estudio tratado en la sección 6 . La información en esta base de datos de términos contempla un pequeño universo de palabras usadas habitualmente en las áreas de las ciencias computacionales, cuya información fue obtenida de fuentes especializadas. Lo anterior no condiciona al usuario a proporcionar textos del ámbito de las ciencias computacionales (caso de estudio) para que la aplicación sea funcional, ya que de no encontrar elementos en la base de datos de términos simplemente el glosario en el material didáctico estará vacío.



Figura 5. Diagrama de flujo de información en la aplicación.

\section{Resultados}

A manera de ejemplificar su desarrollo y prueba, la aplicación incluye una serie de lecciones ejemplo bajo el ámbito de las ciencias computacionales, tomando como referencia algunos tópicos comunes de la computación presentes en los programas de estudio de diferentes instituciones educativas de nivel superior en México (véase Tabla 1). Los tópicos seleccionados fueron: Programación, Redes de Computadoras, Sistemas Operativos, Base de Datos, Ingeniería de Software e Inteligencia Artificial. 


\begin{tabular}{|c|c|c|c|c|c|c|c|c|c|c|}
\hline Institución & Plan de Estudios & $\begin{array}{l}\text { 등 } \\
\text { 은 } \\
\text { 능 } \\
\text { 임 }\end{array}$ & 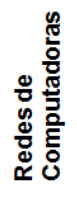 & 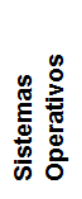 & 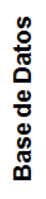 & 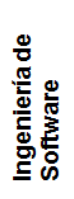 &  & $\begin{array}{l}\text { d } \\
\text { 흘 } \\
\text { 흘 } \\
\text { 흥 }\end{array}$ & 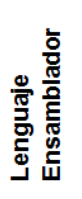 & $\begin{array}{l}\text { 흥 } \\
\text { 엉 } \\
\text { 흔 }\end{array}$ \\
\hline $\begin{array}{l}\text { Benemérita Universidad } \\
\text { Autónoma de Puebla }\end{array}$ & Ingeniería en Computación & $x$ & $x$ & $x$ & $x$ & $x$ & & & $x$ & $\mathrm{X}$ \\
\hline $\begin{array}{l}\text { Benemérita Universidad } \\
\text { Autónoma de Puebla }\end{array}$ & Ciencias de la Computación & $x$ & $x$ & $x$ & $x$ & $x$ & $x$ & $\mathrm{X}$ & $x$ & $\mathrm{x}$ \\
\hline Instituto Politécnico Nacional & Ingeniería en Computación & $x$ & $x$ & $x$ & $x$ & $x$ & $x$ & $x$ & & \\
\hline $\begin{array}{l}\text { Universidad Autónoma Benito } \\
\text { Juárez de Oaxaca }\end{array}$ & Ingeniería en Computación & $\mathrm{x}$ & $x$ & $\mathrm{x}$ & $x$ & $\mathrm{x}$ & $x$ & $\mathrm{X}$ & & $\mathrm{X}$ \\
\hline $\begin{array}{l}\text { Universidad del Istmo, } \\
\text { Campus Tehuantepec }\end{array}$ & Ingeniería en Computación & $\mathrm{x}$ & $x$ & $\mathrm{x}$ & $x$ & $x$ & $x$ & $\mathrm{X}$ & $x$ & $\mathrm{X}$ \\
\hline $\begin{array}{l}\text { Universidad del Valle de } \\
\text { México }\end{array}$ & $\begin{array}{l}\text { Ingeniería en Sistemas } \\
\text { Computacionales }\end{array}$ & $\mathrm{x}$ & $x$ & $x$ & $X$ & & $x$ & & $x$ & $\mathrm{X}$ \\
\hline $\begin{array}{l}\text { Universidad Nacional } \\
\text { Autónoma de México }\end{array}$ & Ciencias de la Computación & $\mathrm{X}$ & $\mathrm{X}$ & $x$ & $\mathrm{X}$ & $\mathrm{x}$ & $x$ & $\mathrm{X}$ & & \\
\hline \multirow[t]{2}{*}{$\begin{array}{l}\text { Universidad Nacional } \\
\text { Autónoma de México }\end{array}$} & Ingeniería en Computación & $x$ & $x$ & $x$ & $x$ & $\mathrm{X}$ & $x$ & $x$ & & $x$ \\
\hline & Total: & 8 & 8 & 8 & 8 & 7 & 7 & 6 & 4 & 6 \\
\hline
\end{tabular}

Tabla 1. Áreas de las ciencias computacionales en planes de estudio de diferentes instituciones en 2015.

Para su evaluación, se aplicaron pruebas considerando métodos de indagación con la finalidad de identificar información y opiniones relevantes en términos del uso, contenido y utilidad de la aplicación por parte de los usuarios. Según Hurtado y Forero (2014), los métodos de indagación son estrategias útiles y sencillas para obtener una primera aproximación de las impresiones del sistema antes de proceder con estrategias más formales concernientes a pruebas de usabilidad. Por lo tanto, se definieron dos estrategias dentro de este marco, una interna en el cual mediante Focus Group con los integrantes de trabajo se consideraron objeto de discusión los aspectos técnicos, pedagógicos y funcionales (véase Figura 6). De esta evaluación interna se definieron mejoras para la provisión de estímulos visuales, la visibilidad, y un diseño responsive.

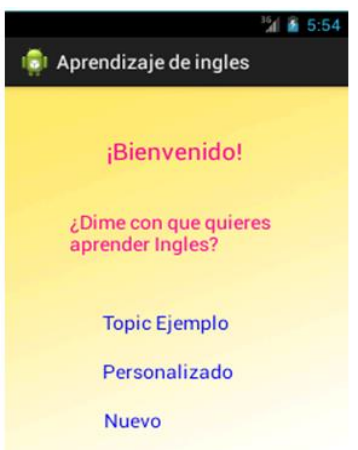

a)

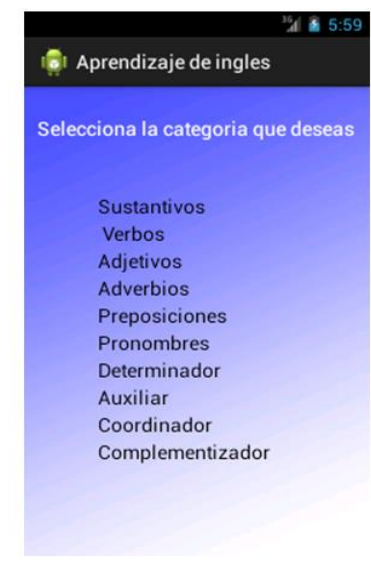

b)

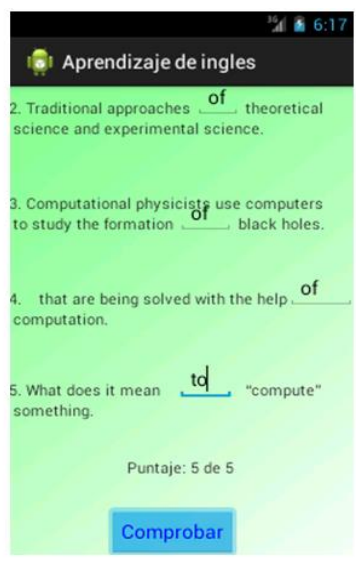

c)

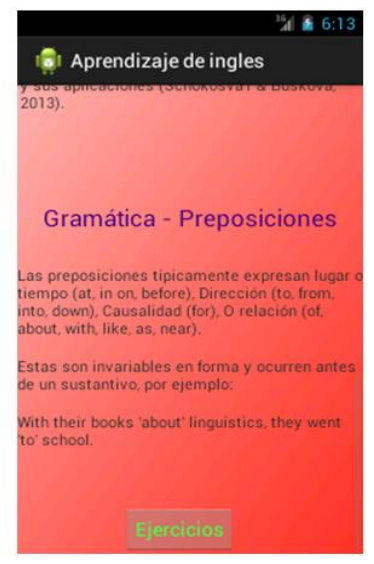

d)

Figura 6. Pantallas en evaluación Focus Group. a) Principal, b) Categorías, c) Ejercicios, b) Lectura-Glosario-Gramática. 
En la Figura 7 se muestran tres pantallas de la aplicación, resultado del proceso de mejora señalado por la evaluación interna. Las Figuras $7 \mathrm{a}, 7 \mathrm{~b}$ y $7 \mathrm{c}$ corresponde a la aplicación en un smartphone con pantalla de 480x800 pixeles. La Figura 7d pertenece a la aplicación en una tablet con resolución de 1024×600 pixeles.

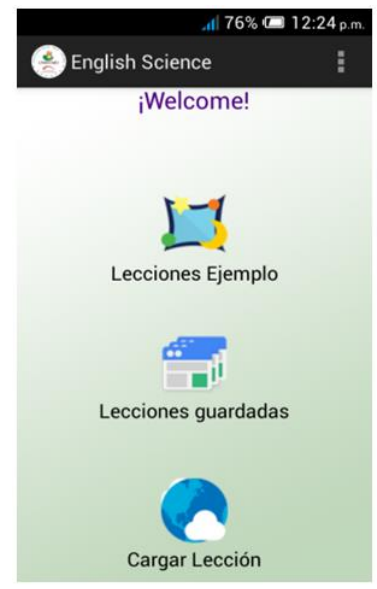

a)

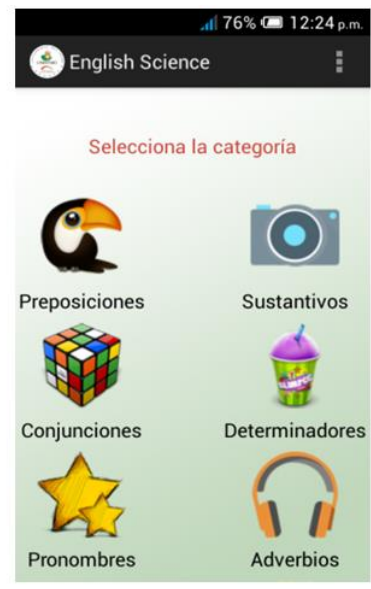

b)



c)

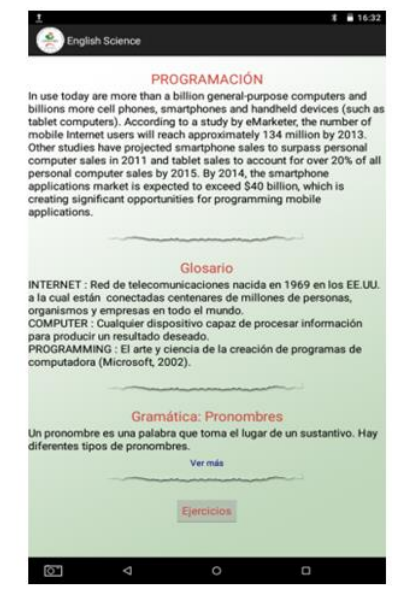

d)

Figura 7. Pantallas. a) Principal, b) Categorías, c) Ejercicios, b) LecturaGlosario-Gramática. Fuente: Elaboración propia.

La evaluación externa se realizó mediante una muestra de 14 alumnos de la carrera de Ingeniería de Computación a los cuales se les proporcionó la aplicación y a través de una encuesta de satisfacción diseñada (véase Figura 8) se captaron una serie de puntos relevantes en términos de uso, contenido y utilidad que se describen a continuación:

- El $86 \%$ de los encuestados aseguran tener un nivel regular o bueno de inglés. De esta forma, se confirma que la población tiene conocimientos básicos del idioma, por lo que podrán observar a la aplicación como una herramienta de apoyo.

- El 93\% considera que la aplicación es fácil de utilizar, el 7\% la cataloga como muy fácil, no hay registro que indique una dificultad para el manejo de la aplicación (véase Figura 9a).

- El $79 \%$ piensa que los contenidos relacionados con las ciencias computacionales son buenos o muy buenos. El $21 \%$ los califica de regulares. Los contenidos no son catalogados como malos, a sabiendas que la población (estudiantes de computación) deberá presentar un mínimo interés (véase Figura 9b).

- El $71 \%$ califica como útil o muy útil la aplicación. No hay registros que indiquen la que aplicación no presente utilidad alguna (véase Figura 9c).

- El $43 \%$ de los encuestados dijeron no estar dispuesto a comprar la aplicación, pero sin embargo algunos comentaron que la usarían si esta fuera gratis. El 57\% consideraría comprar la aplicación. 

Encuesta de satisfacción
1. ¿Cuál considera que es su nivel de inglés?
$\square$ Muy bueno Bueno
Regular
$\square$ Malo
2. El uso de la aplicación le resultó Muy fácil $\square$ Fácil
3. ¿Cómo calificaría los contenidos de la aplicación? Muy buenos Buenos Regulares
4. ¿Qué grado de utilidad le otorgaría a la aplicación? Regular

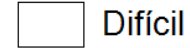
5. ¿Usted compraría esta aplicación? S Si Tal vez Incluya al reverso sus comentarios.



Figura 8. Encuesta de satisfacción.

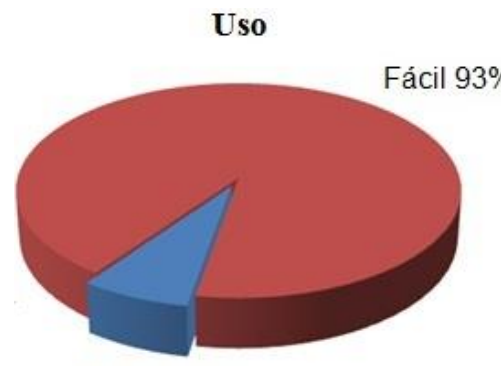

Muy fácil $7 \%$

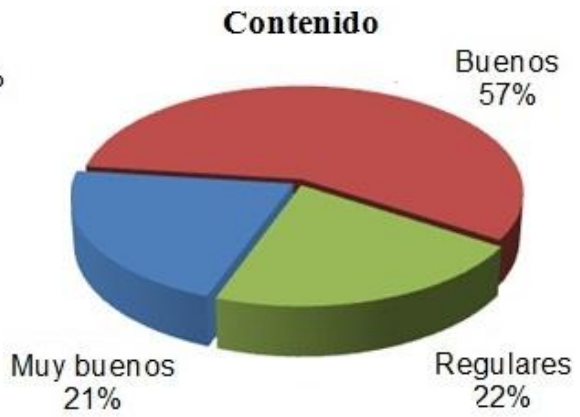

b)

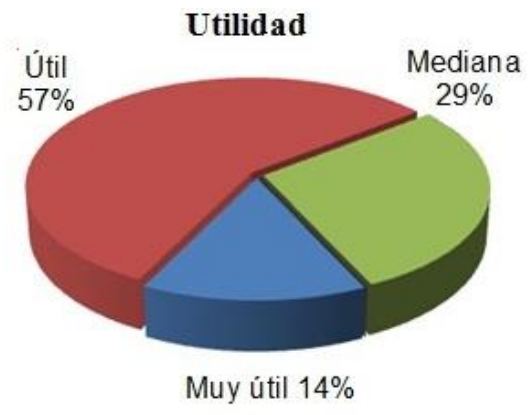

c)

Figura 9. Evaluación de la aplicación. a) Uso, b) Contenido, c) Utilidad.

\section{Conclusiones}

Los cambios en el entorno representan una característica inherente del cómputo móvil, lo cual hace adecuados a los dispositivos móviles para el desarrollo de sistemas basados en el contexto. La aplicación presentada evidencia el uso de la lectura de interés como elemento del perfil de usuario y por tanto de su contexto para fomentar el autoaprendizaje. Así, a un médico le podrá resultar más atractiva una lección de inglés que le hablará de enfermedades, y a un joven aquella que le hable de la serie de televisión del momento. La aplicación permite la adaptación de los materiales didácticos mediante la incorporación de información presente en un archivo de texto.

El trabajo presentado busca situar el interés del usuario por un tipo de lectura en particular, como una herramienta para romper la barrera que representa el inglés, debido a los frecuentes escenarios en el que los estudiantes que cursan sus estudios universitarios tras al menos seis años de cursos de inglés, aún muestren fuertes carencias en conceptos básicos del idioma. 
En el aspecto funcional, el uso de una metodología común para la enseñanza del idioma inglés como lo son las "categorías" o "partes de la oración", facilitó la generación de material didáctico. No obstante la enorme complejidad que presentan los sistemas lingüísticos.

El caso de estudio considerado (ciencias computacionales), verificó la factibilidad del uso textos de interés del usuario como base para el desarrollo de aplicaciones relacionadas con la enseñanza. Conforme a la evaluación preliminar la aplicación muestra ser útil e intuitiva, no obstante resulta adecuado en un trabajo futuro realizar pruebas de usabilidad para estimar la aceptación del producto.

\section{Referencias}

Abowd, G. D., Dey, A. K., Brown, P. J., Davies, N., Smith, M., \& Steggles, P. (1999). Towards a better understanding of context and context-awareness. In Proceedings of the 1st International Symposium on Handheld and Ubiquitous Computing (pp. 304-307). London, UK: Springer-Verlag. doi: 10.1007/3-54048157-5_29

Calderón, D. (2015). Introducción al reporte Sorry y al aprendizaje del inglés en México. México: Mexicanos Primero. Recuperado de http://www.mexicanosprimero.org/images/stories/sorry/discurso-davidcalderon-sorry.pdf

Consejo de Europa (2002). Marco Común Europeo de Referencia para las Lenguas: Aprendizaje, Enseñanza, Evaluación. Madrid, España: Instituto Cervantes. Recuperado de http://cvc.cervantes.es/ensenanza/biblioteca_ele/marco/cvc_mer.pdf

Cuello, J., \& Vittone, J. (2013). Diseñando apps para móviles. Catalina Duque Giraldo. Recuperado de http://appdesignbook.com/es/contenidos/

Ertmer, P., \& Newby, T. (1993). Conductismo, cognitivismo y constructivismo: una comparación de los aspectos críticos desde la perspectiva del diseño de instrucción. Performance improvement quarterly, 6 (4), 50-72.

Fernández, R. (2001). Glosario básico inglés-español para usuarios de Internet (4a ed.). Barcelona, España: Asociación de Técnicos de Informática. Recuperado de http://www2.ati.es/novatica/glosario/glointv4.pdf

Gómez, J. E., Hernández, V. L., \& Morales, M. A. (2015). Arquitectura interactiva como soporte al aprendizaje situado en la enseñanza de la ingeniería. Educación en Ingeniería, 10 (20), 88-97. 
Hurtado, L. L., \& Forero, J. A. (2014). Metodología de evaluación de usabilidad de interfaces humano-máquina. Tecnura, Edición especial 2014, 103-113. doi: http://dx.doi.org/10.14483/udistrital.jour.tecnura.2014.SE1.a08

Jardines, F. J. (2010). Comparación de la educación a distancia con la educación presencial: modelos de educación, diseños instruccionales y rendimiento académico de los alumnos. InnOvaciOnes de NegOciOs, 7 (2). 293314.

Kolangade, O. (2013). A Context-based framework for mobile applications (Master's thesis). Retrieved from http://scholarworks.rit.edu/theses/5530/

Lemus, M. E., Duran, K., \& Martínez, M. (2008). El nivel de inglés y su problemática en tres universidades de México geográficamente distantes. En Memorias del IV Foro Nacional de Estudios en Lenguas (pp. 243-251). Quintana Roo, México: Departamento de Lengua y Educación.

Microsoft (2002). Computer Dictionary (5th ed.). Redmond, Washington: Microsoft Press.

Peña, A. (ed.). (2016). Mobile, Ubiquitous, and Pervasive Learning: Fundaments, Applications, and Trends. Switzerland: Springer.

Remacha, S. (2008). Infotech, English for computer users, Student's Book (4th ed.). Cambridge, UK: Cambridge University Press.

UNESCO (2013). Directrices de la UNESCO para las políticas de aprendizaje móvil. Organización de las Naciones Unidas para la Educación, la Ciencia y la Cultura. Recuperado http://unesdoc.unesco.org/images/0021/002196/219662S.pdf

UNESCO (s.f.). El aprendizaje móvil. Organización de las Naciones Unidas para la Educación, la Ciencia y la Cultura. Recuperado de http://www.unesco.org/new/es/unesco/themes/icts/m4ed/

Stephens, R. (2013). Essential Algorithms: A Practical Approach to Computer Algorithms. Indianapolis, IN: Wiley.

Sun, L., Hou, J., Hu, X., \& Al-mekhlafi, K. (2015). A Context-based Support System of Mobile Chinese Learning for Foreigners in China. Procedia Computer Science, 60, 1396-1405. doi: 10.1016/j.procs.2015.08.215

Van Gelderen, E. (2010). An Introduction to the Grammar of English (Revised ed.). Amsterdam, The Netherlands: John Benjamins Publishing Company. 
Wanumen, L. F., Cavanzo, G.A., \& Guevara, J.C. (2015). Plataforma de enseñanza aprendizaje de conceptos básicos de astronomía basada en un modelo de adaptación sensible al contexto y al perfil del usuario de Facebook. Horizontes Pedagógicos, 17 (2), 76-87.

Zheng, M., Cheng, S.H., \& XU, Q. (2016). Context-Based Mobile User Interface. Journal of Computer and Communications, 4, 1-9. doi: 10.4236/jcc.2016.49001

\section{Notas Biográficas:}

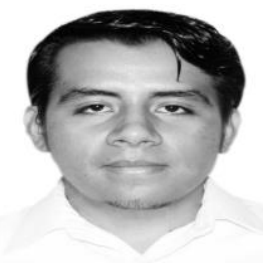

Osiris Montero Ríos es estudiante de Ingeniería en Computación en la Universidad del Istmo. Participó en la Feria Nacional de Ciencia e Ingenierías fase nacional en 2015. Sus áreas de interés incluyen: el cómputo móvil, las bases de datos, y la programación en diferentes lenguajes y entornos.

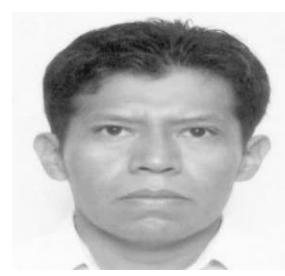

Francisco Aguilar Acevedo es Ingeniero en Electrónica por la Universidad Tecnológica de la Mixteca y Maestro en Ciencias en Ingeniería Mecatrónica por el Centro Nacional de Investigación y Desarrollo Tecnológico. Actualmente es Profesor-Investigador de tiempo completo adscrito a la carrera de Ingeniería en Computación en la Universidad del Istmo, y miembro activo del cuerpo académico de Cómputo Aplicación. Sus áreas de interés incluyen: la inteligencia artificial, la robótica, los sistemas embebidos y la instrumentación electrónica.

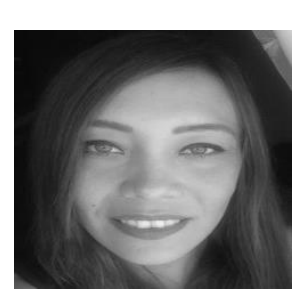

Guadalupe Toledo Toledo es Ingeniera en Sistemas Computacionales por el Instituto Tecnológico de Tuxtepec, y Maestra en Computación Aplicada por parte del Laboratorio Nacional de Informática Avanzada. Actualmente es ProfesorInvestigador de tiempo completo adscrito a la carrera de Ingeniería en Computación en la Universidad del Istmo. Sus áreas de interés se centran en el desarrollo de software y prototipos didácticos con aplicación en ingeniería y computación, aplicación de evaluaciones de usabilidad a productos de software centrados en el usuario y la integración del cómputo aplicado en la solución de problemas multidisciplinares. 


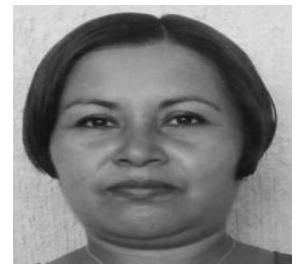

Silvia Reyes Jiménez es Licenciada en Administración y Maestra en Administración y Gestión de Negocios por el Instituto Tecnológico de Oaxaca. Actualmente es Profesor-Investigador de tiempo completo adscrito a la carrera de Ingeniería en Computación en la Universidad del Istmo. Su principal área de investigación es la gestión del conocimiento.

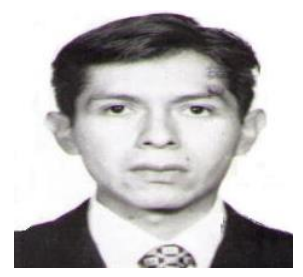

Daniel Pacheco Bautista es ingeniero en electrónica por el Instituto Tecnológico de Oaxaca, Maestro en Ciencias con especialidad en diseño de circuitos integrados por el Instituto Nacional de Astrofísica, Óptica y Electrónica, y Doctor en ingeniería biomédica por la Universidad Popular Autónoma del Estado de Puebla, Actualmente es Profesor-Investigador adscrito a la carrera de Ingeniería en Computación en la Universidad del Istmo. Sus líneas de investigación incluyen: Arquitectura de computadoras y Lógica reconfigurable, Osciladores controlados por voltaje y Circuito de amarre de fase en VLSI, y plataformas alternativas para el ensamble de secuencias cortas de ADN.

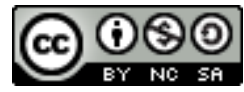

Esta obra está bajo una licencia de Creative Commons Reconocimiento-NoComercial-Compartirlgual 2.5 México.

\footnotetext{
1 Teléfono móvil, teléfono básico o feature phone es un término aplicado a teléfonos móviles con características limitadas respecto a los smartphone.

2 De las competencias lingüísticas, la aplicación se centra en el desarrollo de las competencias léxicas; las cuales comprenden el conocimiento del vocabulario de una lengua y la capacidad para utilizarlo (Consejo de Europa, 2002).
} 